\title{
The receptor CD44 is associated with systemic insulin resistance and proinflammatory macrophages in human adipose tissue
}

\author{
Li Fen Liu ${ }^{1}$ - Keiichi Kodama ${ }^{2,3} \cdot$ Ke Wei $^{1} \cdot$ Lorna L. Tolentino $^{4} \cdot$ Okmi Choi $^{4}$ • \\ Edgar G. Engleman ${ }^{4}$ - Atul J. Butte ${ }^{2,3}$ - Tracey McLaughlin ${ }^{1}$
}

Received: 30 September 2014 / Accepted: 7 April 2015 /Published online: 9 May 2015

(C) Springer-Verlag Berlin Heidelberg 2015

\begin{abstract}
Aims/hypothesis Proinflammatory immune cell infiltration in human adipose tissue is associated with the development of insulin resistance. We previously identified, via a gene expression-based genome-wide association study, the cellsurface immune cell receptor CD44 as a functionally important gene associated with type 2 diabetes. We then showed that, compared with controls, $C d 44$ knockout mice were protected from insulin resistance and adipose tissue inflammation during diet-induced obesity. We thus sought to test whether CD44 is associated with adipose tissue inflammation and insulin resistance in humans.

Methods Participants included 58 healthy, overweight/ moderately obese white adults who met predetermined criteria for insulin resistance or insulin sensitivity based on the modified insulin-suppression test. Serum was collected from 43 participants to measure circulating concentrations of CD44.
\end{abstract}

Li Fen Liu and Keiichi Kodama contributed equally to this work.

Electronic supplementary material The online version of this article (doi:10.1007/s00125-015-3603-y) contains peer-reviewed but unedited supplementary material, which is available to authorised users.

Tracey McLaughlin

tmclaugh@stanford.edu

1 Division of Endocrinology, Department of Medicine, Stanford University, 300 Pasteur Drive, Rm S025, Stanford, CA 94305-5103, USA

2 Division of Systems Medicine, Department of Pediatrics, Stanford University School of Medicine, Stanford, CA, USA

3 Lucile Packard Children's Hospital, Palo Alto, CA, USA

4 Department of Pathology, Stanford University School of Medicine, Stanford, CA, USA
Subcutaneous adipose tissue was obtained from 17 participants to compare $C D 44$, its ligand osteopontin (OPN, also known as $S P P 1)$ and pro-inflammatory gene expression. CD44 expression on adipose tissue macrophage (ATM) surfaces was determined by flow cytometry.

Results Serum CD44 concentrations were significantly increased in insulin-resistant (IR) participants. CD44 gene expression in subcutaneous adipose tissue was threefold higher in the IR subgroup. The expression of $O P N, C D 68$ and IL6 was also significantly elevated in IR individuals. $C D 44$ gene expression correlated significantly with $C D 68$ and $I L 6$ expression. CD44 density on ATMs was associated with proinflammatory M1 polarisation.

Conclusions/interpretation CD44 and OPN in human adipose tissue are associated with localised inflammation and systemic insulin resistance. This receptor-ligand pair is worthy of further research as a potentially modifiable contributor to human insulin resistance and type 2 diabetes.

Keywords Adipose tissue macrophage · Alternatively activated macrophage M2 $\cdot$ Classical phenotype M1 $\cdot$ CD 44 receptor $\cdot$ Insulin resistance

$\begin{array}{ll}\text { Abbreviations } \\ \text { ATM } & \text { Adipose tissue macrophages } \\ \text { eGWAS } & \text { Gene expression-based GWAS } \\ \text { GWAS } & \begin{array}{l}\text { Genome-wide association study } \\ \text { CLS }\end{array} \\ & \begin{array}{l}\text { Crown-like structure ELISA enzyme-linked } \\ \text { immunoassay }\end{array} \\ \text { IS } & \text { Insulin sensitive } \\ \text { IR } & \text { Insulin resistant } \\ \text { MFI } & \text { Mean fluorescent intensity } \\ \text { MCP-1 } & \text { Monocyte chemoattractant protein-1 } \\ \text { OPN } & \text { Osteopontin }\end{array}$


qRT-PCR Quantitative real-time PCR

SSPG Steady-state plasma glucose

\section{Introduction}

While the link between obesity and insulin resistance has been firmly established, the causal basis of this association is unclear. In humans, the total number of macrophages increases in adipose tissue in proportion to BMI and adipose cell diameter [1], and polarisation towards a proinflammatory state (M1) is associated with insulin resistance as measured by the HOMA [2]. In BMI-matched overweight/obese adults, expression of inflammatory genes is significantly higher in insulin-resistant (IR) individuals than in those who are insulin sensitive (IS) [3]. Genetic association studies have revealed a great number of genes consistently associated with obesity and type 2 diabetes [4]. However, these type-2-diabetesassociated genes do not fully elucidate the mechanisms underlying the progression of adipose inflammation and insulin resistance. We recently performed a gene expression-based genome-wide association study (eGWAS) in which we carried out a meta-analysis of genome-wide gene expression data related to type 2 diabetes, and identified $C D 44$ as the top candidate gene functionally linked to type 2 diabetes [4].

CD44 encodes a cell-surface glycoprotein, an immune cell receptor, which is involved in inflammatory cell migration and activation. CD44 is significantly upregulated in white adipose tissue in murine obesity $[4,5]$, and the protein receptor has been shown to be highly expressed on adipose tissue macrophages (ATMs) in humans and mice [4-7]. Multiple studies have shown that knockout of $C d 44$ and/or antibody neutralisation of CD44 in mice prevents macrophage infiltration and activation in white adipose tissue during high-fat feeding, and prevents obesity-induced insulin resistance [4-7]. These studies implicate CD44 as playing a causal role in both inflammation and insulin resistance in model systems. In humans, there is scant data but we have previously shown that serum CD44 levels were positively correlated with HOMA-IR and $\mathrm{HbA}_{1 \mathrm{c}}$ in Japanese individuals [4] and CD44 gene expression has been detected by others in subcutaneous adipose tissue in humans $[6,8]$.

Various ligands for CD44 have been identified, including osteopontin (OPN; also known as secreted phosphoprotein 1). OPN has been shown to mediate obesity-associated insulin resistance in mice, with a high-fat diet leading to increased Opn expression in ATMs and knockout of Opn, decreasing both macrophage infiltration into adipose tissue and insulin resistance $[9,10]$. Interestingly, our eGWAS analysis revealed that $C d 44$ mRNA expression level was positively correlated with the $O p n$ (also known as Spp 1) mRNA expression level in obese mice, suggesting that CD44 and OPN may be closely related in obese adipose tissue. Little data on OPN exists in humans, but a small study in six individuals who underwent bariatric surgery showed dramatic and significant decreases in both $C D 44$ and $O P N$ expression in subcutaneous adipose tissue as a result of massive weight loss [8].

Clearly these provocative studies in mice and preliminary studies in humans warrant further evaluation as to the potential role of CD44 and its ligand, OPN, in mediating human inflammation and insulin resistance. We tested the hypothesis that adipose tissue CD44 would be increased in IR compared with similarly obese IS individuals and that it would correlate with inflammatory activity in adipose tissue. To explore whether OPN might play a role in stimulating CD44-mediated inflammation, we also quantified the latter in human adipose tissue.

\section{Methods}

Participants A total of 58 participants were studied, comprised of two separate cohorts. Cohort 1 had fasting serum samples taken for measurement of CD44 and cohort 2 had adipose tissue biopsies for measurement of gene expression and cell-surface markers to evaluate $C D 44$ and inflammation in adipose tissue. Those with $C D 44$ expression in adipose tissue were included in the serum CD44 cohort. All participants were healthy, overweight/moderately obese adults with BMI $25-36.9 \mathrm{~kg} / \mathrm{m}^{2}$ who were weight stable, non-diabetic (defined as fasting plasma glucose $<6.99 \mathrm{mmol} / \mathrm{l}(126 \mathrm{mg} / \mathrm{dl})$ ) and free from major organ disease or inflammatory conditions. Exclusions included prior bariatric surgery or liposuction, pregnancy, use of glucose-lowering or weight-loss drugs, tobacco use, and use of immune/inflammation-modulating medications.

Measurement of insulin-mediated glucose uptake Insulinmediated glucose uptake was quantified using the octreotidemodified insulin-suppression test as originally described and validated [11, 12]. Briefly, after an overnight fast, participants were infused for $180 \mathrm{~min}$ with octreotide, insulin and glucose, according to body surface area. At steady state (150-180 min), plasma insulin concentrations are similar for all individuals (417 pmol/1 $[60 \mu \mathrm{U} / \mathrm{ml}]$ ), and steady-state plasma glucose (SSPG) concentration provides a direct measure of insulinmediated glucose uptake in response to an infused glucose load; the higher the SSPG concentration, the more insulin resistant the individual. Because SSPG is a continuous measure, to obtain clear separation of groups, insulin resistance was defined as SSPG $>8.05 \mathrm{mmol} / \mathrm{l}(145 \mathrm{mg} / \mathrm{dl})$, insulin sensitivity was defined as SSPG $<6.66 \mathrm{mmol} / 1(120 \mathrm{mg} / \mathrm{dl})$ and those with mid-range SSPG levels were excluded. The study was approved by the Stanford University Human Subjects Committee and all participants gave written, informed consent.

Subcutaneous adipose tissue biopsy and isolation of stromal vascular component After an overnight fast, under sterile 
conditions and local anaesthesia, participants underwent subcutaneous periumbilical adipose tissue biopsies by scalpel incision as previously described [3]. Adipose tissue was cleaned of blood and visible connective tissue and washed with PBS buffer. A portion of adipose tissue was flash frozen on liquid nitrogen for measurement of gene expression. Isolation of adipocytes and stromal vascular cells from remaining tissue was performed via collagenase digestion using a modification of the original protocol of Rodbell [13]. Adipose tissue was mechanically minced and transferred to digestion buffer containing collagenase I, $1 \mathrm{mg} / \mathrm{ml}, 30 \mathrm{~min}$ at $37^{\circ} \mathrm{C}$ in a shaking water bath. The cell suspension was then filtered through a $250 \mu \mathrm{m}$ nylon mesh and centrifuged for $5 \mathrm{~min}$ at $700 \mathrm{~g}$ at room temperature. The resulting cell pellet was collected by further centrifugation at $700 \mathrm{~g}$ for $5 \mathrm{~min}$ and then incubated in erythrocyte lysis buffer for $10 \mathrm{~min}$ at $37^{\circ} \mathrm{C}$. PBS with $2 \%$ BSA was added to the cell suspension following the erythrocyte lysis procedure.

Flow cytometry to quantify macrophage phenotype A single-cell suspension of mononuclear cells from fresh adipose tissue was assayed by multicolour flow cytometry for frequency and phenotype of macrophages using LSRII flow cytometry (BD Biosciences, San Jose, CA, USA) and FACSDiva software (BD, San Jose, CA, USA). Analysis was done using FlowJo software (FlowJo, LLC, Ashland, OR, USA). Isolated cells were stained with monoclonal antibodies purchased from either BD Biosciences and/or BioLegend. (San Diego, CA, USA). Total macrophages were defined as $\mathrm{CD} 45^{+} \mathrm{HLADR}^{+} \mathrm{CD} 14^{+}$ cells. Subsets of interest were further characterised as M1 $\left(\mathrm{CD}_{4} 5^{+} \mathrm{HLADR}^{+} \mathrm{CD} 14^{+} \mathrm{CD} 206^{+} \mathrm{CD} 11 \mathrm{c}^{+}\right)$cells, M2 $\left(\mathrm{CD} 45^{+} \mathrm{HLADR}^{+} \mathrm{CD} 14^{+} \mathrm{CD} 206^{+} \mathrm{CD} 11 \mathrm{c}^{-}\right)$cells and adipose tissue monocytes (CD206 ${ }^{-}$DD11 $\left.{ }^{+}\right)$[2]. The mean fluorescent intensity (MFI) of surface marker CD44 was also measured from each subset of interest. Lineage population (CD3, CD20 and CD56) were excluded from these analyses.

Circulating concentration of CD44 and OPN Fasting serum human sCD44std (standard soluble CD44) was measured in a total of 42 samples by using a quantitative ELISA (Human CD44 instant ELISA, eBioscience San Diego, CA, USA). Fasting plasma human OPN was measured in a total of 35 samples, in which the circulating CD44 was also measured by using a quantitative ELISA (Human osteopontin quantikine ELISA; R\&D Systems, Minneapolis, MN, USA).

Quantitative real-time PCR for gene expression Expression levels of CD44, IL6, CD68 and OPN genes in abdominal subcutaneous adipose tissue were determined by using quantitative real-time PCR (qRT-PCR). Total RNA was extracted from frozen adipose tissue using TriZol (Invitrogen, Carlsbad, CA, USA) and the Adipose Tissue RNAeasy kit (Qiagen, Valencia, CA, USA) according to the manufacturers' instructions. cDNA was synthesised from total RNA using High Capacity RNA-to-cDNA kit (Applied Biosystems, Carlsbad, CA, USA). Gene expression was quantified using Taqman primers/probes (Applied Biosystems). A threshold cycle $\left(C_{t}\right.$ value) was obtained from each amplification curve, and a $\Delta C_{t}$ value calculated by subtracting the $C_{t}$ value for GAPDH from the $C_{t}$ value for each sample. A $\Delta \Delta C_{t}$ value, representing relative gene expression, was then calculated by subtracting the $\Delta \mathrm{C}_{\mathrm{t}}$ value of a single IS individual (control) [14].

Immunohistochemistry Formalin-fixed paraffin-embedded sections were stained with antibodies against CD68 (KP1; ab955, 1:100; Abcam San Francisco, CA, USA), CD3 (N1580, 1:1; DAKO), CD19 (250585, 1:100; ABBIOTEC), and CD44 (IM7; 553131, 1:100; BD Pharmingen, San Jose, CA, USA). 3,3'-Diaminobenzidine (DAB) was used as chromogen and haematoxylin as counterstain. We created digitised images with a BIOQUANT Image Analysis System (BIOQUANT Image Analysis, Nashville, TN, USA).

Statistical analysis All variables were normally distributed. Dependent variables of interest (CD44, OPN, IL6 and CD68 gene expression, and circulating CD44 and OPN) in IR vs IS subgroups were compared by ANCOVA, with adjustment for BMI. Correlational analyses used multiple linear regression analysis with adjustment for BMI. Data were expressed as mean $\pm \mathrm{SE}$, and $p<0.05$ was considered statistically significant.

\section{Results}

Demographic, clinical and laboratory characteristics Circulating CD44 concentrations were measured in 43 obese participants (cohort 1) and gene expression and cell-surface markers for macrophage phenotype in 17 participants (cohort 2). Demographic, clinical and laboratory characteristics of the IS and IR groups are compared in Table 1. SSPG concentration by design was significantly higher in the IR group than in the IS group in both cohorts (cohort 1: 12.21 \pm 2.33 vs $5.28 \pm 1.27 \mathrm{mmol} / 1, p<0.001$; cohort $2: 12.34 \pm 2.61 \mathrm{vs}$ $5.07 \pm 1.35 \mathrm{mmol} / 1, p<0.001)$. Also, by design, the IR and IS groups were not statistically significantly different in terms of BMI in either cohort. Although there were no significant differences in age, race, total cholesterol, triacylglycerol and LDL-cholesterol concentrations between IR and IS group in either cohort, the IR group had significantly higher fasting glucose and lower HDL-cholesterol in comparison with the IS group (Table 1).

Circulating CD44 concentrations in obese humans To determine the association of receptor CD44 and insulin 
Table 1 Demographic and clinical characteristics of the study participants

\begin{tabular}{|c|c|c|c|c|c|c|}
\hline \multirow[t]{2}{*}{ Characteristic } & \multicolumn{3}{|c|}{ Cohort 1 (serum) } & \multicolumn{3}{|c|}{ Cohort 2 (adipose tissue biopsy) } \\
\hline & IS $(n=22)$ & $\mathrm{IR}(n=21)$ & $p$ value & IS $(n=8)$ & $\operatorname{IR}(n=9)$ & $p$ value \\
\hline Age (years) & $55 \pm 8$ & $58 \pm 5$ & 0.19 & $52.1 \pm 9.0$ & $56.8 \pm 4.7$ & 0.20 \\
\hline Sex (female/male) & $13 / 9$ & $9 / 11$ & 0.36 & $5 / 3$ & $5 / 4$ & 0.77 \\
\hline Race (white/African-American) & $20 / 0$ & $17 / 2$ & 0.28 & $8 / 0$ & $9 / 0$ & 0.81 \\
\hline BMI $\left(\mathrm{kg} / \mathrm{m}^{2}\right)$ & $30.5 \pm 3.0$ & $31.7 \pm 2.7$ & 0.18 & $30.9 \pm 5$ & $32.9 \pm 4.6$ & 0.32 \\
\hline $\mathrm{SSPG}(\mathrm{mmol} / \mathrm{l})$ & $5.28 \pm 1.27$ & $12.21 \pm 2.33$ & $<0.001$ & $5.07 \pm 1.35$ & $12.34 \pm 2.61$ & $<0.001$ \\
\hline Glucose $(\mathrm{mmol} / \mathrm{l})$ & $5.44 \pm 0.49$ & $5.76 \pm 0.54$ & 0.08 & $5.44 \pm 0.36$ & $5.71 \pm 0.35$ & 0.181 \\
\hline Cholesterol (mmol/l) & $4.71 \pm 0.94$ & $4.56 \pm 0.61$ & 0.64 & $5.34 \pm 1.92$ & $4.94 \pm 0.91$ & 0.632 \\
\hline Triacylglycerol (mmol/l) & $1.04 \pm 0.28$ & $1.46 \pm 1.14$ & 0.31 & $1.0 \pm 1.16$ & $1.46 \pm 0.06$ & 0.06 \\
\hline HDL-cholesterol (mmol/l) & $1.55 \pm 0.52$ & $1.15 \pm 0.21$ & 0.04 & $1.55 \pm 0.31$ & $1.12 \pm 0.24$ & 0.02 \\
\hline LDL-cholesterol (mmol/1) & $2.78 \pm 0.68$ & $2.73 \pm 0.59$ & 0.8 & $3.6 \pm 1.88$ & $3.14 \pm 0.95$ & 0.604 \\
\hline
\end{tabular}

Values are presented as $n / n$ or means $\pm \mathrm{SD}$

resistance, we measured CD44 serum levels in 43 moderately obese participants. Serum CD44 levels were significantly higher in the IR group than in the IS group $(323 \pm 91 \mathrm{ng} / \mathrm{ml}$ vs $264 \pm 88 \mathrm{ng} / \mathrm{ml}$, respectively, $p=0.045$ ) (Fig. 1). In addition, we measured OPN concentration in plasma samples in 35 moderately obese participants who also had CD44 levels measured. Plasma OPN levels were significantly higher in the IR group than in the IS group $(48.6 \pm 19.8 \mathrm{ng} / \mathrm{ml}$ vs $27.9 \pm$ $21.9 \mathrm{ng} / \mathrm{ml}$, respectively, $p=0.017$ ) (ESM Fig. 1).

$C D 44, O P N$ and inflammatory gene expression in adipose tissue To extend the serum findings of CD44 and to further explore the potential role of OPN in the proinflammatory state in human obese participants, CD44, OPN, CD68 and IL6 mRNA expression was measured by qRT-PCR in subcutaneous adipose tissue obtained from the participants in cohort 2 (Table 1). CD44 gene expression in subcutaneous adipose tissue was threefold higher in the IR group $(p=0.006)$ (Fig. 2a). IL6, $C D 68$ and $O P N$ were also expressed at significantly higher levels in IR than in IS individuals (Fig. 2b-d). Correlational analysis between gene expression (Fig. 3)

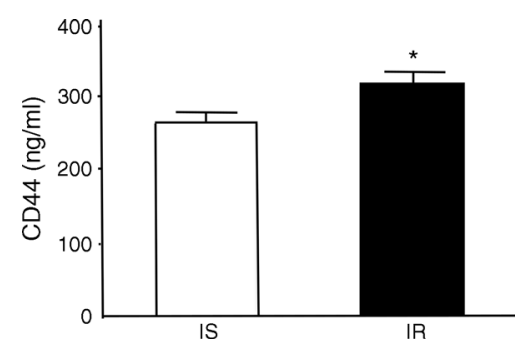

Fig. 1 Circulating CD44 concentrations in white participants. CD44 levels were evaluated by ELISA in serum of IS $(n=22)$ and IR participants $(n=21)$. Results are expressed as mean \pm SE. ${ }^{*} p<0.05$ vs IS revealed significant correlations between $C D 44$ and $C D 68$ $(p=0.01)$ and $I L 6$ ( $p=0.039)$ (Fig. 3b, c), with a modest but non-significant correlation between $C D 44$ and $O P N(p=0.18)$ (Fig. 3a) and between $O P N$ and $C D 68$ (not shown) $(p=0.09)$.

We explored within our cohort whether there was an association between BMI and CD44, but found no association in either serum or adipose tissue (univariate correlation between BMI and CD44 gene expression, $n=17, r=0.08, p=0.76$; correlation between BMI and serum CD44, $n=43, r=0.11$, $p=0.48$ ).

Analysis of ATMs by flow cytometry To analyse the polarisation of ATMs, flow cytometry analysis was performed on ATMs isolated from the stromal vascular cell fraction of subcutaneous adipose tissue. Total macrophages, defined as $\mathrm{CD} 45^{+} \mathrm{HLADR}^{+} \mathrm{CD} 14^{+}$, were further characterised as M1 $\left(\mathrm{CD} 11 \mathrm{c}^{+} \mathrm{CD} 206^{+}\right)$and $\mathrm{M} 2\left(\mathrm{CD} 11 \mathrm{c}^{-} \mathrm{CD} 206^{+}\right)$, whereas $\mathrm{CD} 14^{+} \mathrm{CD} 206^{-} \mathrm{CD} 11 \mathrm{c}^{+}$cells were identified as adipose tissue monocytes (Fig. 4a). Macrophages sorted according to proinflammatory (M1) phenotype (vs anti-inflammatory [M2] phenotype) showed significantly higher expression of CD44, as measured by MFI (CD44/M1 vs CD44/M2, $p=0.0017, n=15$ ) (Fig. 4b).

Immunohistochemical localisation of CD44 in human adipose tissue We further investigated the composition of cell types and the location/expression of CD44 in crown-like structures. We conducted an immunohistochemical analysis for CD68 (macrophage marker), CD3 (T cell marker), CD19 (B cell marker) and CD44 in human adipose tissue. We found that most infiltrating cells in fat tissues were stained with anti-CD68 and anti-CD44 antibodies, suggesting that many inflammatory cells in the crown-like structures are macrophages and are positive for CD44 (Fig. 5). 
Fig. 2 Relative expression of $C D 44, I L 6, C D 68$ and $O P N$ in subcutaneous adipose tissue of IS vs IR participants. Relative expression of CD44 (a), IL6 (IS, $n=7$; IR, $n=5$ ) (b), $C D 68$ (c) and $O P N$ mRNA (d) was analysed by real-time quantitative PCR in the subcutaneous adipose tissue of obese participants (IS, $n=8$; IR, $n=9)$. Data are presented as mean \pm SE for IS vs IR groups, ${ }^{*} p<0.05$ vs IS
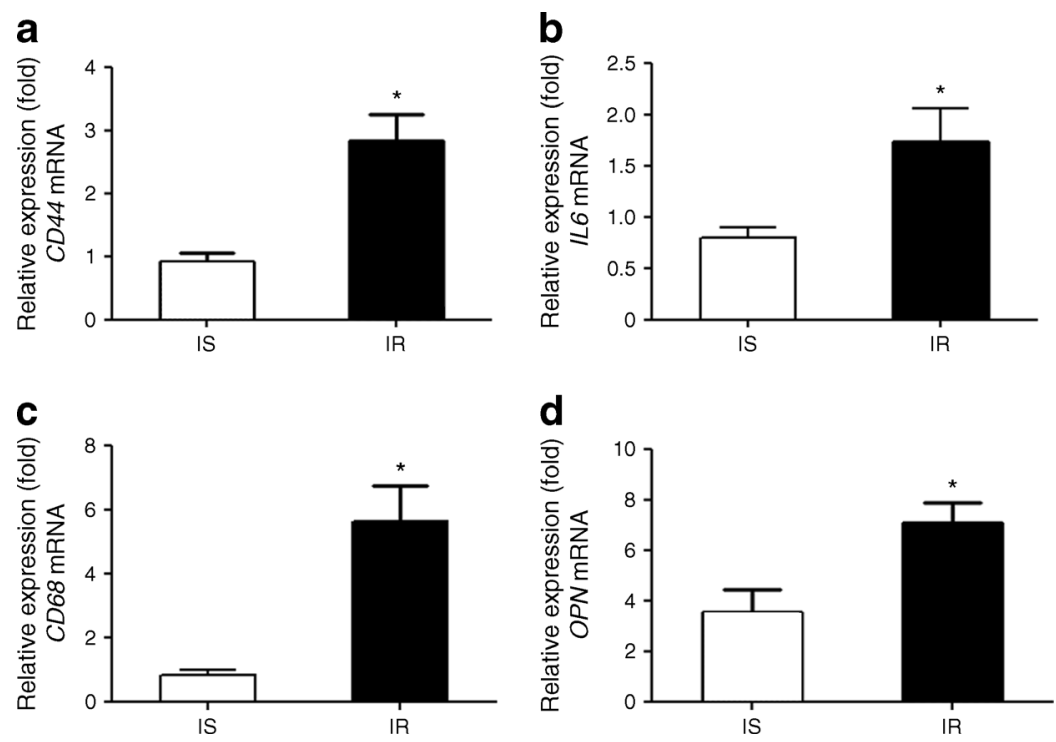

\section{Discussion}

The results of this study highlight a new, potentially important pathway of inflammation and insulin resistance in humans by demonstrating that the immunological cell-surface receptor CD44, and its ligand, OPN, are highly expressed in human adipose tissue and are significantly increased in IR as compared with IS overweight/moderately obese individuals. Furthermore, CD44 expression was significantly higher on the surface of ATMs bearing markers of classical vs alternative activation, and gene expression of $C D 44$ correlated with that of IL6 and CD68, lending further support for a role of CD44 in inciting adipose tissue inflammation.

The current results extend the findings of two small studies on CD44 in human adipose tissue. One showed that CD44 was abundantly expressed in both subcutaneous and omental adipose tissue in morbidly obese individuals and that it decreased significantly after surgically induced weight loss in six individuals with follow-up at 2 years [8]. The other study demonstrated that CD44, while expressed in obese adipose tissue, was only expressed in non-adipocyte cell subfractions, including ATMs and stromal vascular subfractions [6]. However, neither study evaluated the relationship between CD44 and insulin-mediated glucose uptake at the cellular or systemic level, inflammation or macrophage activation, although among six individuals who underwent adipose tissue biopsy 2 years after bariatric surgery the expression of $C D 44$ correlated significantly with that of $C D 68$ [8]. To our knowledge, our study is the first to directly associate CD44 in human adipose tissue with insulin resistance, quantified by a gold-standard test and macrophage activation markers. Further, because study participants were matched for BMI, we were able to isolate the relationship between CD44 and insulin resistance without confounding by obesity. Thus, the current findings lend support to the notion that physical characteristics of adipose tissue, and not simply fat mass per se, contribute to the development of insulin resistance.

Soluble CD44 shed from cell surfaces exists in normal human serum. We demonstrated that serum CD44 was associated with insulin resistance in 43 overweight and obese individuals. These results support the observations in adipose tissue and ATMs, and are consistent with our findings from a a

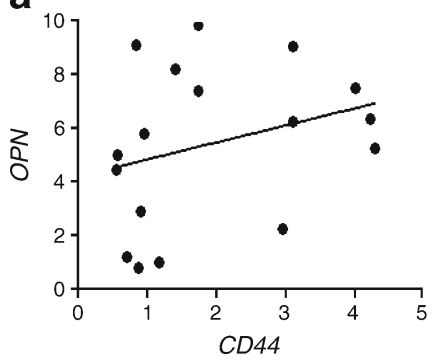

b

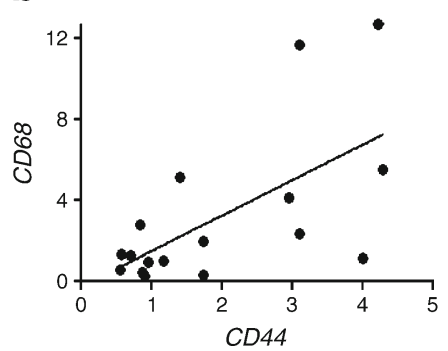

C

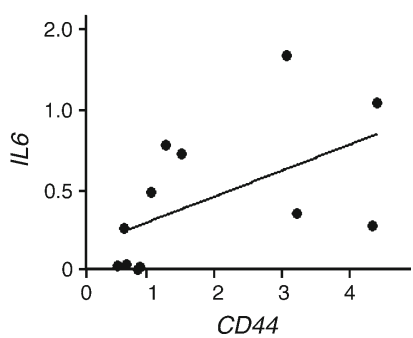

Fig. 3 Correlations between expression of $C D 44$ and $O P N, C D 68$ and IL 6 mRNA in human adipose tissue. Correlations between the expression levels of $C D 44$ mRNA and $O P N(\mathbf{a}, r=0.34, p=0.18), C D 68(\mathbf{b}, r=0.61$, $p=0.01)$ and $I L 6$ mRNA (c, $r=0.60, p=0.039)$ were analysed using
Spearman's rank correlation test. Relative expression of $C D 44, O P N$, $C D 68$ and IL6 mRNA was determined by qRT-PCR in the subcutaneous adipose tissue of obese human participants 


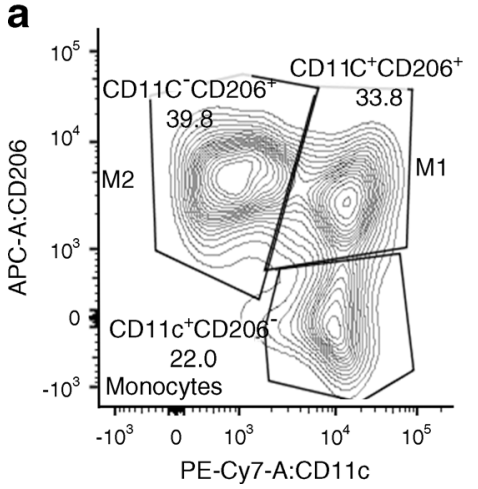

Fig. 4 CD44 density in human subcutaneous adipose tissue M1 and M2 macrophages. (a) Flow cytometry phenotype of gated M1

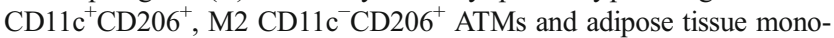
cytes $\left(\mathrm{CD} 11 \mathrm{c}^{+} \mathrm{CD} 206^{-}\right)$. ATM cells can be separated into three distinct subpopulations when stained for CD11c and CD206. Yaxis, CD206 cells stained with APC-A (allophycocyanin-a). (b) CD44 density in phenotype of M1 (CD11 $\left.\mathrm{c}^{+} \mathrm{CD} 206^{+}\right)$vs M2 (CD11 $\left.{ }^{-} \mathrm{CD} 206^{+}\right)$ATMs as determined

prior study in non-diabetic Japanese adults showing that a simple index of insulin resistance, as estimated by HOMA, was correlated with serum CD44 concentrations [4]. These findings suggest that CD44 protein may be released from IR adipose tissues into circulation in humans. This may indicate the significance of CD44 as a possible novel biomarker for insulin resistance and low-grade adipose tissue inflammation.

While associative in nature, the current findings in humans extend the observations made in mice, which strongly suggest that CD44 plays a causal role in both adipose tissue inflammation and insulin resistance. We previously demonstrated an 11-fold increase in adipose tissue expression of $C d 44$ in obese as compared with lean mice [4]. Furthermore, knockout of Cd44 prevented the development of obesity-induced insulin resistance and glucose intolerance [4, 7], as well as inflammation in adipose tissue and liver $[15,16]$. Recently, we also found that CD44 is strongly expressed on ATMs in dietinduced obese mice, and antibody neutralisation of CD44 suppressed visceral adipose tissue inflammation and reduced hyperglycaemia and insulin resistance as effectively as metformin and pioglitazone [7]. In addition, RT-PCR showed that expression of M1 macrophage activation markers (e.g. CD68, TNF- $\alpha$, monocyte chemoattractant protein-1 [MCP-1] and IL-6) was downregulated in adipose tissue of mice treated with anti-CD44 monoclonal antibody [7]. These data in mice support the findings of a growing body of in vitro studies demonstrating that CD44 plays a role in mediating adhesion, b

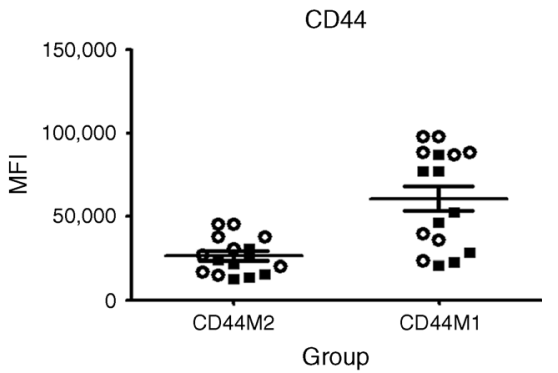

by flow cytometry. Total macrophages were characterised as $\mathrm{CD} 5^{+} \mathrm{HLADR}^{+} \mathrm{CD} 14^{+}$cells by flow cytometry. Macrophages sorted according to proinflammatory (M1) phenotype vs anti-inflammatory (M2) phenotype showed significantly higher expression of CD44, measured by MFI ( $p=0.0017, n=15)$. Macrophages from IR and IS participants are indicated by circles and squares, respectively

migration and inflammatory cytokine secretion by $\mathrm{T}$ lymphocytes and macrophages [17-24]. Consistent with the finding that CD44 on the cell surface is associated with M1 macrophage activation by FACS, immunohistochemical localisation of CD44 in human adipose tissue reveals that CD44-positive cells are strongly associated with crown-like structures in human adipose tissue. Based on this evidence and our current data, we hypothesise that CD44 is a functionally important molecule on human ATMs, potentiating recruitment and activation and possibly contributing to systemic insulin resistance. Further research into the molecular pathways by which this occurs in human ATMs and adipose cells is merited.

OPN and hyaluronic acid are known ligands for the CD44 receptor. While hyaluronic acid mediates cell aggregation via binding with CD44, OPN, stimulates macrophage migration and inflammatory cytokine secretion primarily via interaction with CD44 receptor [18, 22-24]. While OPN's role in regulating bone remodelling has been well studied, its expression by inflammatory cells and regulation of their function has only recently been addressed. Recent studies show that OPN is highly upregulated in adipose tissue in obese mice and humans $[6,8]$. In mice, but not humans, OPN has been linked to insulin resistance, glucose intolerance and inflammation: high-fat diet in wild-type C57BL/6 mice for 20 weeks led to an eightfold increase in adipose tissue OPN expression as well as increased plasma OPN concentrations, insulin resistance,
Fig. 5 Immunohistochemical analysis of CD44 and immune cell markers (CD68, CD3 and CD19) in subcutaneous fat tissue. Scale bar, $50 \mu \mathrm{m}$

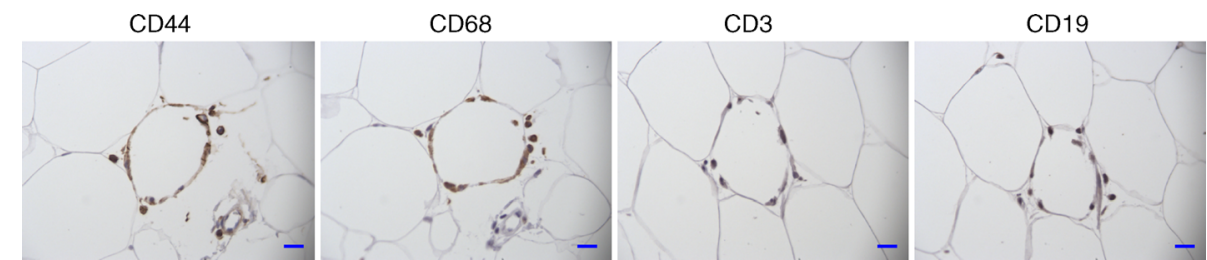


glucose intolerance and adipose tissue inflammation [9]. Opnknockout mice fed the same diet, despite similar weight and body-fat gain, were protected from insulin resistance, glucose intolerance and ATM infiltration [9]. In contrast, neutralisation of OPN prevents obesity-induced inflammation and insulin resistance in mice [25]. While OPN plasma concentrations are increased in obese humans, data are not available for the effect of OPN on insulin resistance in humans. Here we showed that OPN plasma concentrations are higher in IR humans independent of obesity.

While it is now well accepted that macrophages infiltrate subcutaneous and visceral adipose tissue in humans, the stimuli for macrophage infiltration are not well characterised. The only well-characterised ligand-receptor pair to date is MCP-1-C-C motif chemokine receptor type 2 (CCR2), which, as shown by knockout studies, appears to account for some but not all macrophage infiltration into adipose tissue [26]. The described knockout studies for both CD44 and OPN strongly suggest that this ligand-receptor pair may also be important for macrophage recruitment and activation in adipose tissue. The current findings that OPN plasma concentration was higher in IR participants than in IS participants and that OPN expression in human adipose tissue was strongly associated with insulin resistance further supports a role for this CD44 ligand in promoting inflammation and insulin resistance in humans. While its correlation with CD44 expression in human adipose tissue did not reach statistical significance in the current study, in our initial eGWAS study, $O P N$ was not only one of the top candidate genes predictive of type 2 diabetes but it also correlated highly with $C D 44$ [4].

In conclusion, these novel findings strongly suggest that CD44 and OPN may represent an important ligand-receptor pair that determines macrophage infiltration and activation in human adipose tissue and/or systemic insulin resistance. Identification of treatments to modify OPN or CD44, or their interaction, may potentially contribute to the prevention of obesity-induced inflammation and/or insulin resistance.

\footnotetext{
Acknowledgements Grant support for this study was provided by the American Diabetes Association, Translational Research Grant and NIH/ NIDDK 1R01DD080436.
}

Duality of interest The authors declare that there is no duality of interest associated with this manuscript.

Contribution statement LFL and KK conceived and designed experiments, acquired and analysed data and wrote the manuscript. KW, LLT and $\mathrm{OC}$ acquired and analysed data and drafted the manuscript. EGE, AJB and TLM designed the experiment and critically reviewed and revised the manuscript. All authors approved the final version of the manuscript to be published. LFL and KK are responsible for the integrity of the work as a whole.

\section{References}

1. Weisberg S, McCann D, Desai M et al (2003) Obesity is associated with macrophage accumulation in adipose tissue. J Clin Invest 112: 1796-1808

2. Wentworth JM, Naselli G, Brown WA et al (2010) Proinflammatory $\mathrm{CD} 11 \mathrm{c}^{+} \mathrm{CD} 206^{+}$adipose tissue macrophages are associated with insulin resistance in human obesity. Diabetes 59: 1648-1656

3. McLaughlin T, Sherman A, Tsao P et al (2007) Enhanced proportion of small adipose cells in insulin-resistant vs insulin-sensitive obese individuals implicates impaired adipogenesis. Diabetologia 50:1707-1715

4. Kodama K, Horikoshi M, Toda K et al (2012) Expression-based genome-wide association study links the receptor CD44 in adipose tissue with type 2 diabetes. Proc Natl Acad Sci U S A 109:70497054

5. Kiefer FW, Zeyda M, Todoric J et al (2008) Osteopontin expression in human and murine obesity: extensive local upregulation in adipose tissue but minimal systemic alterations. Endocrinology 149: 1350-1357

6. Zeyda M, Gollinger K, Todoric J et al (2011) Osteopontin is an activator of human adipose tissue macrophages and directly affects adipocyte function. Endocrinology 152:2219-2227

7. Kodama K, Toda K, Morinaga S et al (2015) Anti-CD44 antibody treatment lowers hyperglycemia and improves insulin resistance, adipose inflammation, and hepatic steatosis in diet-induced obese mice. Diabetes 64:867-875

8. Bertola A, Deveaux V, Bonnafous S et al (2009) Elevated expression of osteopontin may be related to adipose tissue macrophage accumulation and liver steatosis in morbid obesity. Diabetes 58: $125-133$

9. Nomiyama T, Perez-Tilve D, Ogawa D et al (2007) Osteopontin mediates obesity-induced adipose tissue macrophage infiltration and insulin resistance in mice. J Clin Invest 117:2877-2888

10. Gomez-Ambrosi J, Catalan V, Ramirez B et al (2007) Plasma osteopontin levels and expression in adipose tissue are increased in obesity. J Clin Endocrinol Metab 92:3719-3727

11. Pei D, Jones CN, Bhargava R, Chen YD, Reaven GM (2004) Evaluation of octreotide to assess insulin-mediated glucose disposal by the insulin suppression test. Diabetologia 37:843-845

12. Greenfield M, Loberne L, Kraemer F, Tobey T, Reaven G (1981) Assessment of insulin resistance with the insulin suppression test and euglycemic clamp. Diabetes 30:387-392

13. Rodbell M, Metabolism of isolated fat cells (1964) Effects of hormones on glucose metabolism and lipolysis. J Biol Chem 239:375-380

14. Livak KJ, Schmittgen TD (2001) Analysis of relative gene expression data using real-time quantitative PCR and the $2^{-\Delta \Delta \mathrm{C}_{\mathrm{t}}}$ method. Methods 25:402-408

15. Kang HS, Liao G, DeGraff LM et al (2013) CD44 plays a critical role in regulating diet-induced adipose inflammation, hepatic steatosis, and insulin resistance. PLoS One 8:e58417

16. Egan CE, Daugherity EK, Rogers AB, Abi Abdallah DS, Denkers EY, Maurer KJ (2013) CCR2 and CD44 promote inflammatory cell recruitment during fatty liver formation in a lithogenic diet fed mouse model. PLoS One 8:e65247

17. Cuff CA, Kothapalli D, Azonobi I et al (2001) The adhesion receptor $\mathrm{CD} 44$ promotes atherosclerosis by mediating inflammatory cell recruitment and vascular cell activation. J Clin Invest 108: 1031-1040

18. Singh M, Ananthula S, Milhorn DM, Krishnaswamy G, Singh K (2007) Osteopontin: a novel inflammatory mediator of cardiovascular disease. Front Biosci 12:214-221 
19. DeGrendele HC, Estess P, Sieelman MH et al (1997) Requirement for CD44 in activated T cell extravasation into an inflammatory site. Science 278:672-675

20. Huet S, Groux H, Caillou B, Valentin H, Prieur AM, Bernard A (1989) CD44 contributes to T cell activation. J Immunol 143:798801

21. McKee CM, Penno MB, Cowman M, Bao C, Novle PW (1996) Hyaluronan (HA) fragments induce chemokine gene expression in alveolar macrophages. The role of HA size and CD44. J Clin Invest 98:2403-2413

22. Giachelli CM, Lombardi D, Johnson RJ, Murry CE, Almeida M (1998) Evidence for a role of osteopontin in macrophage infiltration in response to pathological stimuli in vivo. Am J Pathol 152:353358
23. Zhu B, Suzuki K, Goldberg HA et al (2004) Osteopontin modulates CD44-dependent chemotaxis of peritoneal macrophages through G-protein-coupled receptors: evidence of a role for an intracellular form of osteopontin. J Cell Physiol 198:155-167

24. Weber GF, Ashkar S, Glimcher MJ, Cantor H (1996) Receptorligand interaction between CD44 and osteopontin (Eta-1). Science 271:509-512

25. Kiefer FW, Zeyda M, Gollinger K et al (2010) Neutralization of osteopontin inhibits obesity-induced inflammation and insulin resistance. Diabetes 59:935-946

26. Kanda H, Tateya S, Tamori Y (2006) MCP-1 contributes to macrophage infiltration into adipose tissue, insulin resistance, and hepatic steatosis in obesity. J Clin Invest 116:1494-1505 